\title{
Therapeutic potential of hepatocyte growth factor against cerebral ischemia (Review)
}

\author{
WEN ZENG ${ }^{1}$, RONG JU ${ }^{1}$ and MENG MAO M $^{2,3}$ \\ ${ }^{1}$ Department of Neonatology, Chengdu Women's and Children's Central Hospital, Chengdu, Sichuan 610031; \\ ${ }^{2}$ Department of Pediatrics, West China Second University Hospital, Sichuan University, Chengdu, Sichuan 610041; \\ ${ }^{3}$ Key Laboratory of Obstetric \& Gynecologic and Pediatric Diseases and Birth Defects of Ministry of Education, \\ Chengdu, Sichuan 610041, P.R. China
}

Received March 21, 2014; Accepted November 24, 2014

DOI: $10.3892 /$ etm.2014.2133

\begin{abstract}
The effective treatment for cerebral ischemia has not yet been established. Hepatocyte growth factor (HGF) is a potent pleiotropic cytokine that is involved in cell and tissue regeneration, including in the central nervous system. Studies have demonstrated that an exogenous administration of HGF protects brain tissue from ischemic damage. In response to binding to the receptor c-Met, HGF activates the downstream signaling pathways (including the phosphatidylinositol 3-kinase/Akt, Ras/MAPK and signal transducer and activator of transcription pathways) which leads to various cellular responses involved in angiogenesis, glial scar formation, anti-apoptosis and neurogenesis. The purpose of this review is to summarize the present understanding of the therapeutic potential of HGF in cerebral ischemia.
\end{abstract}

\section{Contents}

1. Introduction

2. HGF and its receptor c-Met

3. HGF as a potential therapy for cerebral ischemia

4. Challenges

5. Conclusion

\section{Introduction}

Cerebral ischemia causes an irreversible and neurodegenerative disorder that may lead to progressive dementia and cognitive

Correspondence to: Professor Meng Mao, Department of Pediatrics, West China Second University Hospital of Sichuan University, 20 People of South Road, Chengdu, Sichuan 610041, P.R. China

E-mail:dffmmao@126.com

Key words: hepatocyte growth factor, c-Met, cerebral ischemia, angiogenesis, antifibrosis, neurogenesis deterioration. However, no effective treatment has been established yet to prevent brain injury following ischemia.Hepatocyte growth factor (HGF), also referred to as Scatter factor, was first identified and purified from plasma and serum as a potent mitogen for hepatocytes in 1984 by Nakamura et al (1-3). Subsequently, HGF was identified in several other organs, including the lungs, kidneys and heart, as well as in blood vessels (4). In the 1990s, the wide distribution of HGF was identified in the central neural system (CNS) $(5,6)$. HGF is now a well-known potent pleiotropic cytokine that is involved in mitogenesis, motogenesis, morphogenesis, angiogenesis and anti-apoptosis in a variety of cells, and tissue regeneration in several organs (7-9). HGF has been reported to improve the neurological sequelae by reducing the infarct volume following a stroke (10-12). This suggested that HGF should be one of the most potent growth factors for treating brain ischemia. In this review, we primarily focus on the role of HGF as a potential therapy for ischemic brain damage and the possible mechanisms.

\section{HGF and its receptor c-Met}

HGF was first identified as a mitogenic protein for rat hepatocytes in 1984 (13), and was thereafter purified from rat platelets, human plasma and rabbit plasma (14). In 1989, cDNA for human HGF was cloned and the primary structure of HGF was clarified, by which HGF was identified as a novel growth factor with unique structural characteristics (3). HGF is secreted as a single-chain, inactive polypeptide by mesenchymal cells and is cleaved to its active extracellular form by a number of proteases. The active HGF is a heterodimer composed of a $69-\mathrm{kDa} \alpha$-chain and a $34-\mathrm{kDa} \beta$-chain. The $\alpha$-chain contains an $\mathrm{N}$-terminal hairpin domain followed by four kringle domains, and the $\beta$-chain contains a serine protease-like domain with no enzymatic activity $(3,15)$.

The proto-oncogene product receptor tyrosine kinase c-Met is the only known receptor for HGF. The human Met (HGF receptor) gene is located on chromosome 7q21-q31. c-Met is synthesized as a $170-\mathrm{kDa}$ glycosylated precursor protein that is cleaved into a $50-\mathrm{kDa} \alpha$-chain and a $140-\mathrm{kDa}$ $\beta$-chain that are linked by disulfide bonds (16).

In response to ligand (HGF) binding, c-Met undergoes autophosphorylation on two tyrosine residues (Y1234 and 
Y1235) within the activation loop of the tyrosine kinase domain, which regulate the intrinsic kinase activity of c-Met. Phosphorylation of Y1349 and Y1356 near the C-terminus of c-Met forms a multifunctional signal transducer docking site (Y1349VHVX3Y1356VNV) that binds a number of substrates containing Src homology-2 (SH2) domains, including growth factor receptor-bound protein 2 (Grb2), Gab1, phosphatidylinositol 3-kinase (PI3K), phospholipase C- $\gamma$ (PLC- $\gamma$ ), Shp2 and $\operatorname{Scr}(17,18)$. This leads to the activation of downstream signaling pathways including the PI3K/Akt, Ras/MAPK and signal transducer and activator of transcription (STAT) pathways (19-21). Activation of the HGF/c-Met signaling pathway has been shown to lead to various cellular responses including proliferation, angiogenesis, wound healing, tissue regeneration, scattering, motility, invasion and branching morphogenesis.

\section{HGF as a potential therapy for cerebral ischemia}

Angiogenesis. Angiogenesis was first described as a vital factor in tumor growth in 1971 (22) and then defined as the formation of new vessels sprouting from pre-existing capillaries in the pathological or physiological processes in adult tissue (23). It may be highly regulated by the action of growth factors, proteolytic enzymes or other extracellular matrix factors that stimulate the growth of endothelial cells. With the increased interest in angiogenesis and more in-depth research, it is considered that angiogenesis plays a significant role in minimizing tissue injury as the collateral blood flow supplies oxygen and energy substrate to the ischemic area. Therefore, the concept of therapeutic angiogenesis was proposed and became a new means of therapy, which is the clinical use of growth factors to enhance or promote the development of collateral blood vessels in ischemic tissue (24).

For a long time, attempts to alleviate ischemic cerebral injuries and ameliorate the prognosis have focused on ensuring or improving the survival of neurons, while ignoring the role of angiogenesis. However, the latter might be closely correlated with the survival of neurons following the ischemic insults. Krupinski et al (25) first reported that capillary density was increased in infarcted brain tissue of patients who had survived acute ischemic stroke for up to several weeks, indicating that increased angiogenesis is beneficial for longer survival of patients. Previous animal studies have revealed that inhibition of vascular endothelial cell proliferation promoted neural cell death, and that the application of proangiogenic regulator eased the ischemic injuries $(26,27)$. Indeed, for treating limb ischemia and myocardial infarction, proangiogenic regulators became a new means of therapy. Angiogenesis should be a potent therapy for stroke patients through increasing the cerebral blood flow. Other studies also demonstrated that vascular endothelial growth factor (VEGF), a notable proangiogenic factor (28), increases vascular density, reduces brain damage and improves neurological deficits (29-32), suggesting that angiogenic therapy may be helpful for ischemic brain injury.

As a potent angiogenic molecule, HGF mediates angiogenesis primarily through direct actions on vascular endothelial cells. Studies have demonstrated that HGF and c-Met are expressed and functional in vascular endothelial cells of various origins, including neuromicrovascular endothelial cells (33). Shang et al observed that HGF significantly amplified the angiogenesis following middle cerebral artery occlusion (34). Date et al $(35,36)$ also reported that HGF could prevent the learning and memory dysfunction induced by cerebral ischemia by protecting the endothelial cells against injury.

The molecular mechanisms of the angiogenic activity of HGF may be strongly associated with the E-twenty-six (ETS) pathway, since the ETS family plays a significant role in regulating gene expression in response to the multiple developmental and mitogenic signals $(37,38)$. By activating the specific receptor, c-Met, HGF also induces DNA synthesis and proliferation of vascular endothelial cells through MAPK/ERK and STAT3 pathway activation $(39,40)$.

In addition to the proliferative effects, HGF also protects endothelial cells against apoptosis or cell death induced by various detrimental insults, including hypoxia and serum deprivation. However, the signal pathways that mediate the protective effects are not fully known. Ma et al (41) revealed that the MAPK/ERK and Akt pathway may mediate the HGF-induced survival of endothelial cells. Other experiments indicate that HGF protects endothelial cells against hypoxic injury associated with inhibition of p38 MAPK and Bid/Bax as well as increased expression of Bcl-2 or Bcl-xl $(42,43)$.

It is known that matrix degradation and remodeling are indispensable in angiogenesis, and allow endothelial cell migration and invasion $(44,45)$. HGF induces or upregulates the expression and synthesis of matrix metalloproteinases (MMPs) and urokinase-type plasminogen activator by vascular endothelial cells, and accelerates endothelial cell invasion into the extracellular matrix during angiogenesis $(46,47)$. HGF also stimulates dissociation and migration of vascular endothelial cells, which may occur through the modulating action of HGF on the vascular endothelial cadherin (48).

In addition, HGF regulates angiogenesis through interacting with other well-known angiogenic regulators. It has been shown that HGF induces VEGF expression at both the mRNA and protein levels, which might be regulated by MAKP, PI3K, PKC and Sp1, a modulator of the VEGF promoter $(40,48)$. Further studies identified that essential transcription factor ets-1 was upregulated by HGF and contributed to HGF-induced VEGF expression $(38,49)$. HGF and VEGF play synergistic effects in promoting vascular endothelial cell survival with augmented expression of the anti-apoptotic genes Bcl-2 and A1 (48). Moreover, HGF is capable of downregulating the expression of thrombospondin 1, which negatively regulates angiogenesis $(50,51)$.

Furthermore, compared with other angiogenic regulators including VEGF, HGF has noted advantages in promoting angiogenesis, as follows: a) it does not disrupt the blood brain barrier (BBB); b) it does not increase cerebral edema; c) it does not cause vascular inflammation; and d) it has anti-thrombosis ability $(35,36,52,53)$. VEGF is a major mediator of angiogenesis as well as being a strong vascular permeability factor and a notable stroke-related pathogenic factor for the formation of brain edema (54-56), which limits its therapeutic applications. HGF avoids these disadvantages. It is suggested that HGF-mediated prevention of endothelial cell injury and maintenance of the tight junctional proteins in the endothelial cells may be a possible mechanism for the protective effect of HGF against the disruption of the $\mathrm{BBB}$ and the prevention of cerebral edema (36). 
Anti-apoptosis and neurogenetic effects. Since mature neurons cannot duplicate, it is essential to maintain their survival to improve the outcome of cerebral ischemia. The roles of excitatory amino acid receptor activation, calcium overload, nitric oxide and oxidative stress are well established in the pathogenesis of ischemic brain damage (57). Studies have reported that HGF notably decreases the infarct volume of ischemic brain tissue and protects neurons from death caused by N-methyl-Daspartate excitotoxicity (11), and it also prevents neuron death by inhibition of apoptosis through the blockade of bax translocation from the cytoplasm to the nucleus $(58,59)$. In addition, HGF stimulates ERK1/2, PI3K/Akt and STAT3 activity in neurons, and then induces the transcription of neuroprotective genes, including bcl-2 and Bcl-xl, protecting neurons from apoptosis following ischemia (39,60-62).

In recent years, there has been a growing interest in the therapeutic potential of stem cells. Stem cells are multipotent and self-renewing cells, so it is believed that they may be beneficial to the outcome of cerebral stroke. There is evidence revealing that transplantation of neural stem cells (NSCs) or mesenchymal stem cells (MSCs) decreases the infarcted area and improves functional outcomes (63-66). HGF promotes the proliferation, differentiation and migration of NSCs and MSCs (67-69). However, the mechanisms involved are still not completely understood. It has been reported that HGF induces the activation of its downstream effectors ERK1/2, p38MAPK and PI3K/Akt, which contribute to the effects of modulating the migration, proliferation and differentiation of NSCs and MSCs, while the regulation is abrogated by specific inhibitors (68-72).

Antifibrosis. Ischemia induces tissue damage to the CNS and activates astrocytes, leading to reactive gliosis, which causes glial scar formation (73). A glial scar presents as a rubbery, tenacious growth-blocking membrane, which consists predominately of reactive astrocytes and chondroitin sulfate proteoglycans, including neurocan and phosphacan $(74,75)$. Although studies indicate that glial scarring serves to repair the $\mathrm{BBB}$, prevent an excessive inflammatory response and limit cellular degeneration following injury, it also has the disadvantage of inhibiting neuronal and axonal regeneration, causing failure in the structural and functional reconstruction of the CNS following injury (75-79). Therefore, inhibiting glial scar formation is a critical issue for nerve regeneration and functional reconstruction.

As a multifunctional cytokine, it was suggested by Ha et al (80) that HGF prevents pathological scar formation in vivo and in vitro. Another study revealed that HGF decreases glial scar formation and scar thickness of the brain pia mater following transient middle cerebral artery occlusion, indicating that HGF is beneficial for ischemic injury due to its antifibrotic ability (34). A possible mechanism is that HGF markedly inhibits the proliferation and migration of astrocytes in the formation process of glial scarring by the sphingosine-1-phosphate pathway, which is closely related to cell proliferation (81).

Aside from the proliferation and activation of astrocytes, several other factors are also associated with the formation of glial scarring, including transforming growth factor- $\beta$ (TGF- $\beta$ ) and extracellular matrix components. Previous studies suggest that HGF plays antifibrotic roles by regulating proteoglycan synthesis. When astrocytes are activated, four classes of proteoglycans are produced by astrocytes, which are closely related to glial scar formation $(73,74,82)$. TGF- $\beta 1$ is a potent fibrogenic protein that has been shown to significantly increase the production of proteoglycans by astrocytes and cause severe astrogliosis $(83,84)$. However, Jeong et al revealed that HGF completely blocked secretion of TGF- $\beta 1$ from activated astrocytes (85). After binding to c-Met, HGF upregulates the activity and protein expression of est-1 (38). est-1 has a DNA-binding domain and activates transcription of genes encoding uPA and various metalloproteases (e.g., MMP-1 and MMP-9) (86). Via the est-1 pathway, HGF plays its role in decreasing glial scar formation.

\section{Challenges}

The clinical use of HGF is quite limited at present for a number of reasons, including lack of effective administration methods and adverse effects. We will discuss these below.

Administration methods. A notable breakthrough has been made in using HGF in the therapy for limb ischemia, which is at the clinical trial stage (87). However, the administration of $\mathrm{HGF}$ as a recombinant protein for CNS disorders is hindered by a number of issues, including the short serum half-life and poor access to the CNS by the systemic route due to the presence of BBB.

Gene therapy may solve the issue of degradation, but the safety and efficiency of the gene carrier must be ensured. Retroviruses are one of the widely used gene carriers, and can integrate the gene into the chromosomes of the target cells (88-91). However, clinical use is limited due to the potential rise of a neoplasm with a retrovirus-based vector (92).

Considering the safety of gene therapies, certain scientists have proposed a new solution: transferring the virus vector into the host cells (e.g., MSCs) and then transferring the cells into the injured organs (93-95). As reported, MSCs have protective effects in cerebral ischemia $(64,93)$. After being transfected with vector encoding HGF gene, increasing the expression of HGF, the protective effects are enhanced $(85,96,97)$. This method also avoids the possible detriment of virus vector and decreases immunity reactions. However, this therapeutic method is still tested on animals and needs considerable research and effort for clinical practice.

Adverse effects of tumorigenesis. Previous studies have demonstrated that HGF plays a role in tumorigenesis through its capability to promote angiogenesis and mitogenesis (98-101). Therefore, if HGF were used to treat ischemic brain injury, the issue of how to decrease or eliminate the risk of tumorigenesis must be taken into consideration. This issue could possibly be solved by selecting the optimal concentration and time point of medication.

\section{Conclusion}

Overall, as a growth factor, HGF has therapeutic potential against cerebral ischemia. Binding to the receptor c-Met, downstream signaling pathways are phosphorylated and 
activated, including the PI3K/Akt, Ras/MAPK and STAT pathways, then HGF is capable of regulating angiogenesis, glial scar formation, neurogenesis and anti-apoptosis, protecting the brain from ischemic insults. Although certain obstacles remain before clinical application of HGF can be achieved, we are of the opinion that through the deepening research these issues will be overcome, bringing benefit to patients with cerebral ischemia.

\section{References}

1. Nakamura T, Nawa K, Ichihara A, Kaise $\mathrm{N}$ and Nishino $\mathrm{T}$ : Purification and subunit structure of hepatocyte growth factor from rat platelets. FEBS Lett 224: 311-316, 1987.

2. Nakamura T, Nawa K and Ichihara A: Partial purification and characterization of hepatocyte growth factor from serum of hepatectomized rats. Biochem Biophys Res Commun 122: 1450-1459, 1984

3. Nakamura T, Nishizawa T, Hagiya M, et al: Molecular cloning and expression of human hepatocyte growth factor. Nature 342: 440-443, 1989

4. Ohmichi $\mathrm{H}$, Matsumoto $\mathrm{K}$ and Nakamura $\mathrm{T}$ : In vivo mitogenic action of HGF on lung epithelial cells: pulmotrophic role in lung regeneration. Am J Physiol 270: L1031-L1039, 1996.

5. Hamanoue M, Takemoto N, Matsumoto K, Nakamura T, Nakajima K and Kohsaka S: Neurotrophic effect of hepatocyte growth factor on central nervous system neurons in vitro. J Neurosci Res 43: 554-564, 1996.

6. Achim C, Katyal S, Wiley C, et al: Expression of HGF and cMet in the developing and adult brain. Brain Res Dev Brain Res 102 299-303, 1997.

7. Sun W, Funakoshi $\mathrm{H}$ and Nakamura T: Overexpression of HGF retards disease progression and prolongs life span in a transgenic mouse model of ALS. J Neurosci 22: 6537-6548, 2002.

8. Zarnegar R and Michalopoulos G: The many faces of hepatocyte growth factor: from hepatopoiesis to hematopoiesis. J Cell Biol 129: 1177-1180, 1995

9. Matsumoto K and Nakamura T: Emerging multipotent aspects of hepatocyte growth factor. J Biochem 119: 591-600, 1996.

10. Miyazawa T, Matsumoto K, Ohmichi H, Katoh H, Yamashima T and Nakamura T: Protection of hippocampal neurons from ischemia-induced delayed neuronal death by hepatocyte growth factor: a novel neurotrophic factor. J Cereb Blood Flow Metab 18: 345-348, 1998.

11. Tsuzuki N, Miyazawa T, Matsumoto K, Nakamura T and Shima K: Hepatocyte growth factor reduces the infarct volume after transient focal cerebral ischemia in rats. Neurol Res 23 : 417-424, 2001.

12. Shimamura M, Sato N, Oshima K, et al: Novel therapeutic strategy to treat brain ischemia: overexpression of hepatocyte growth factor gene reduced ischemic injury without cerebral edema in rat model. Circulation 109: 424-431, 2004.

13. Nakamura T, Nawa K and Ichihara A: Partial purification and characterization of hepatocyte growth factor from serum of hepatectomized rats. Biochem Biophys Res Commun 122: 1450-1459, 1984.

14. Abounader R and Laterra J: Scatter factor/hepatocyte growth factor in brain tumor growth and angiogenesis. Neuro Oncol 7: 436-451, 2005

15. Matsumoto $\mathrm{K}$ and Nakamura $\mathrm{T}$ : Hepatocyte growth factor: molecular structure, roles in liver regeneration, and other biological functions. Crit Rev Oncog 3: 27-54, 1992.

16. Ma PC, Maulik G, Christensen J and Salgia R: c-Met: structure, functions and potential for therapeutic inhibition. Cancer Metastasis Rev 22: 309-325, 2003.

17. Organ SL and Tsao MS: An overview of the c-MET signaling pathway. Ther Adv Med Oncol 3 (Suppl 1): S7-S19, 2011.

18. Lawrence RE and Salgia R: MET molecular mechanisms and therapies in lung cancer. Cell Adh Migr 4: 146-152, 2010.

19. Trusolino L, Bertotti A and Comoglio PM: MET signalling: principles and functions in development, organ regeneration and cancer. Nat Rev Mol Cell Biol 11: 834-848, 2010.

20. Okano J, Shiota G, Matsumoto K, et al: Hepatocyte growth factor exerts a proliferative effect on oval cells through the PI3K/AKT signaling pathway. Biochem Biophys Res Commun 309: 298-304, 2003.
21. Xiao GH, Jeffers M, Bellacosa A, Mitsuuchi Y, Woude GFV and Testa JR: Anti-apoptotic signaling by hepatocyte growth factor/Met via the phosphatidylinositol 3-kinase/Akt and mitogen-activated protein kinase pathways. Proc Natl Acad Sci USA 98: 247-252, 2001.

22. Folkman J: Tumor angiogenesis: therapeutic implications. N Engl J Med 285: 1182-1186, 1971.

23. Folkman J and Shing Y: Angiogenesis. J Biol Chem 267: 10931-10934, 1992.

24. Henry TD: Therapeutic angiogenesis. BMJ 318: 1536-1539, 1999.

25. Krupinski J, Kaluza J, Kumar P, Kumar S and Wang JM: Role of angiogenesis in patients with cerebral ischemic stroke. Stroke 25: 1794-1798, 1994.

26. Shimotake J, Derugin N, Wendland M, Vexler ZS and Ferriero DM: Vascular endothelial growth factor receptor-2 inhibition promotes cell death and limits endothelial cell proliferation in a neonatal rodent model of stroke. Stroke 41: 343-349, 2010.

27. Yang JP, Liu HJ and Liu XF: VEGF promotes angiogenesis and functional recovery in stroke rats. J Invest Surg 23: 149-155, 2010.

28. Ferrara N: Role of vascular endothelial growth factor in the regulation of angiogenesis. Kidney Int 56: 794-814, 1999.

29. Yano A, Shingo T, Takeuchi A, et al: Encapsulated vascular endothelial growth factor-secreting cell grafts have neuroprotective and angiogenic effects on focal cerebral ischemia. J Neurosurg 103: 104-114, 2005.

30. Bellomo M, Adamo EB, Deodato B, et al: Enhancement of expression of vascular endothelial growth factor after adenoassociated virus gene transfer is associated with improvement of brain ischemia injury in the gerbil. Pharmacol Res 48: 309-317, 2003.

31. Harrigan MR,Ennis SR, Masada T and Keep RF: Intraventricular infusion of vascular endothelial growth factor promotes cerebral angiogenesis with minimal brain edema. Neurosurgery 50: 589-598, 2002.

32. Shen F, Su H, Fan Y, et al: Adeno-associated viral vector-mediated hypoxia-inducible vascular endothelial growth factor gene expression attenuates ischemic brain injury after focal cerebral ischemia in mice. Stroke 37: 2601-2606, 2006.

33. Rosen EM, Laterra J, Joseph A, et al: Scatter factor expression and regulation in human glial tumors. Int J Cancer 67: 248-255, 1996.

34. Shang J, Deguchi K, Ohta Y, et al: Strong neurogenesis, angiogenesis, synaptogenesis, and antifibrosis of hepatocyte growth factor in rats brain after transient middle cerebral artery occlusion. J Neurosci Res 89: 86-95, 2011.

35. Date I, Takagi N, Takagi K, et al: Hepatocyte growth factor attenuates cerebral ischemia-induced learning dysfunction. Biochem Biophys Res Commun 319: 1152-1158, 2004.

36. Date I, Takagi N, Takagi K, et al: Hepatocyte growth factor attenuates cerebral ischemia-induced increase in permeability of the blood-brain barrier and decreases in expression of tight junctional proteins in cerebral vessels. Neurosci Lett 407: 141-145, 2006.

37. Morishita R, Aoki M, Hashiya N, et al: Therapeutic angiogenesis using hepatocyte growth factor (HGF). Curr Gene Ther 4: 199-206, 2004.

38. Aoki M, Morishita R, Taniyama Y, et al: Angiogenesis induced by hepatocyte growth factor in non-infarcted myocardium and infarcted myocardium: up-regulation of essential transcription factor for angiogenesis, ets. Gene Ther 7: 417-427, 2000.

39. Nakagami H, Morishita R, Yamamoto K, et al: Mitogenic and antiapoptotic actions of hepatocyte growth factor through ERK, STAT3, and AKT in endothelial cells. Hypertension 37: 581-586, 2001.

40. Dong G, Chen Z, Li ZY, Yeh NT, Bancroft CC and Van Waes C: Hepatocyte growth factor/scatter factor-induced activation of MEK and PI3K signal pathways contributes to expression of proangiogenic cytokines interleukin- 8 and vascular endothelial growth factor in head and neck squamous cell carcinoma. Cancer Res 61: 5911-5918, 2001.

41. Ma P, Tretiakova M, Nallasura V, Jagadeeswaran R, Husain A and Salgia R: Downstream signalling and specific inhibition of c-MET/HGF pathway in small cell lung cancer: implications for tumour invasion. Br J Cancer 97: 368-377, 2007.

42. Yamamoto K, Morishita R, Hayashi S, et al: Contribution of $\mathrm{Bcl}-2$, but not Bcl-xL and Bax, to antiapoptotic actions of hepatocyte growth factor in hypoxia-conditioned human endothelial cells. Hypertension 37: 1341-1348, 2001. 
43. Wang X, Zhou Y, Kim HP, et al: Hepatocyte growth factor protects against hypoxia/reoxygenation-induced apoptosis in endothelial cells. J Biol Chem 279: 5237-5243, 2004.

44. Davis GE and Senger DR: Endothelial extracellular matrix biosynthesis, remodeling, and functions during vascular morphogenesis and neovessel stabilization. Circ Res 97: 1093-1107, 2005.

45. Chang $\mathrm{C}$ and Werb Z: The many faces of metalloproteases: cell growth, invasion, angiogenesis and metastasis. Trends Cell Biol 11: S37-S43, 2001.

46. Wang $\mathrm{H}$ and Keiser JA: Hepatocyte growth factor enhances MMP activity in human endothelial cells. Biochem Biophys Res Commun 272: 900-905, 2000.

47. Bussolino F, Di Renzo M, Ziche M, et al: Hepatocyte growth factor is a potent angiogenic factor which stimulates endothelial cell motility and growth. J Cell Biol 119: 629-641, 1992.

48. Xin X, Yang S, Ingle G, et al: Hepatocyte growth factor enhances vascular endothelial growth factor-induced angiogenesis in vitro and in vivo. Am J Pathol 158: 1111-1120, 2001.

49. Tomita N, Morishita R, Taniyama Y, et al: Angiogenic property of hepatocyte growth factor is dependent on upregulation of essential transcription factor for angiogenesis, ets-1. Circulation 107: 1411-1417, 2003.

50. Zhang YW, Su Y, Volpert OV and Woude GF: Hepatocyte growth factor/scatter factor mediates angiogenesis through positive VEGF and negative thrombospondin 1 regulation. Proc Natl Acad Sci USA 100: 12718-12723, 2003.

51. Scarpino S, Di Napoli A, Taraboletti G, Cancrini A and Ruco LP Hepatocyte growth factor (HGF) downregulates thrombospondin 1 (TSP-1) expression in thyroid papillary carcinoma cells. J Pathol 205: 50-56, 2005.

52. Birukova AA, Alekseeva E, Mikaelyan A and Birukov KG: HGF attenuates thrombin-induced endothelial permeability by Tiam1-mediated activation of the Rac pathway and by Tiam1/ Rac-dependent inhibition of the Rho pathway. FASEB J 21: 2776-2786, 2007.

53. Kaga T, Kawano H, Sakaguchi M, Nakazawa T, Taniyama Y and Morishita R: Hepatocyte growth factor stimulated angiogenesis without inflammation: Differential actions between hepatocyte growth factor, vascular endothelial growth factor and basic fibroblast growth factor. Vascul Pharmacol 57: 3-9, 2012.

54. Yao X, Miao W, Li M, et al: Protective effect of albumin on VEGF and brain edema in acute ischemia in rats. Neurosci Lett 472: 179-183, 2010

55. Wang Y, Kilic E, Kilic, et al: VEGF overexpression induces postischaemic neuroprotection, but facilitates haemodynamic steal phenomena. Brain 128: 52-63, 2005.

56. Zhang ZG, Zhang L, Jiang Q, et al: VEGF enhances angiogenesis and promotes blood-brain barrier leakage in the ischemic brain. J Clin Invest 106: 829-872, 2000.

57. Szydlowska $\mathrm{K}$ and Tymianski M: Calcium, ischemia and excitotoxicity. Cell Calcium 47: 122-129, 2010.

58. Hayashi K, Morishita R, Nakagami H, et al: Gene therapy for preventing neuronal death using hepatocyte growth factor: in vivo gene transfer of HGF to subarachnoid space prevents delayed neuronal death in gerbil hippocampal CA1 neurons. Gene Ther 8: 1167-1173, 2001.

59. Niimura M, Takagi N, Takagi K, et al: Prevention of apoptosisinducing factor translocation is a possible mechanism for protective effects of hepatocyte growth factor against neuronal cell death in the hippocampus after transient forebrain ischemia. J Cereb Blood Flow Metab 26: 1354-1365, 2006.

60. Qiu J, Cafferty WB, McMahon SB and Thompson SW: Conditioning injury-induced spinal axon regeneration requires signal transducer and activator of transcription 3 activation. J Neurosci 25: 1645-1653, 2005.

61. Dziennis S and Alkayed NJ: Role of signal transducer and activator of transcription 3 in neuronal survival and regeneration. Rev Neurosci 19: 341-362, 2008.

62. He F, Wu LX, Shu KX, et al: HGF protects cultured cortical neurons against hypoxia/reoxygenation induced cell injury via ERK $1 / 2$ and PI-3K/Akt pathways. Colloids Surf B Biointerfaces 61: 290-297, 2008.

63. van Velthoven CT, Kavelaars A, van Bel F and Heijnen CJ: Regeneration of the ischemic brain by engineered stem cells: fuelling endogenous repair processes. Brain Res Rev 61: 1-13, 2009.

64. Dharmasaroja P: Bone marrow-derived mesenchymal stem cells for the treatment of ischemic stroke. J Clin Neurosci 16: 12-20, 2009.
65. Lee HJ, Kim KS, Kim EJ, et al: Brain transplantation of immortalized human neural stem cells promotes functional recovery in mouse intracerebral hemorrhage stroke model. Stem Cell 25 1204-1212, 2007.

66. Tang Y, Yasuhara T, Hara K, et al: Transplantation of bone marrow-derived stem cells: a promising therapy for stroke. Cell Transplant 16: 159-169, 2007.

67. Kokuzawa J, Yoshimura S, Kitajima H, et al: Hepatocyte growth factor promotes proliferation and neuronal differentiation of neural stem cells from mouse embryos. Mol Cell Neurosci 24: 190-197, 2003.

68. Forte G, Minieri M, Cossa P, et al: Hepatocyte growth factor effects on mesenchymal stem cells: proliferation, migration, and differentiation. Stem Cells 24: 23-33, 2006.

69. Nicoleau C, Benzakour O, Agasse F, et al: Endogenous hepatocyte growth factor is a niche signal for subventricular zone neural stem cell amplification and self-renewal. Stem Cells 27: 408-419, 2009.

70. Zheng B, Wang C, He L, et al: Neural differentiation of mesenchymal stem cells influences chemotactic responses to HGF. J Cell Physiol 228: 149-162, 2013.

71. Vogel S, Peters C, Etminan N, et al: Migration of mesenchymal stem cells towards glioblastoma cells depends on hepatocytegrowth factor and is enhanced by aminolaevulinic acid-mediated photodynamic treatment. Biochem Biophys Res Commun 431: 428-432, 2013.

72. Kendall SE, Najbauer J, Johnston HF, et al: Neural stem cell targeting of glioma is dependent on phosphoinositide 3-kinase signaling. Stem Cells 26: 1575-1586, 2008.

73. Zhang H, Muramatsu T, Murase A, Yuasa S, Uchimura K and Kadomatsu K: N-Acetylglucosamine 6-O-sulfotransferase-1 is required for brain keratan sulfate biosynthesis and glial scar formation after brain injury. Glycobiology 16: 702-710, 2006.

74. Sofroniew MV: Molecular dissection of reactive astrogliosis and glial scar formation. Trends Neurosci 32: 638-647, 2009.

75. Silver J and Miller J: Regeneration beyond the glial scar. Nat Rev Neurosci 5: 146-156, 2004

76. Fitch MT and Silver J: CNS injury, glial scars, and inflammation: Inhibitory extracellular matrices and regeneration failure. Exp Neurol 209: 294-301, 2008.

77. Wanner IB, Deik A, Torres M, et al: A new in vitro model of the glial scar inhibits axon growth. GLIA 56: 1691-1709, 2008.

78. Voskuhl RR, Peterson RS, Song B, et al: Reactive astrocytes form scar-like perivascular barriers to leukocytes during adaptive immune inflammation of the CNS. J Neurosci 29: 11511-11522, 2009.

79. Rolls A, Shechter R and Schwartz M: The bright side of the glial scar in CNS repair. Nat Rev Neurosci 10: 235-241, 2009.

80. Ha X, Li Y, Lao M, Yuan B and Wu C: Effect of human hepatocyte growth factor on promoting wound healing and preventing scar formation by adenovirus-mediated gene transfer. Chin Med J (Engl) 116: 1029-1033, 2003.

81. Liu C, Wu Z, Shu C, et al: Experimental investigation of HGF inhibiting glial scar in vitro. Cell Mol Neurobiol 31: 259-268, 2011.

82. McKeon RJ, Jurynec MJ and Buck CR: The chondroitin sulfate proteoglycans neurocan and phosphacan are expressed by reactive astrocytes in the chronic CNS glial scar. J Neurosci 19: 10778-10788, 1999.

83. Yin J, Sakamoto K, Zhang H, et al: Transforming growth factor-beta1 upregulates keratan sulfate and chondroitin sulfate biosynthesis in microglias after brain injury. Brain Res 1263: 10-22, 2009.

84. Schachtrup C, Ryu JK, Helmrick MJ, et al: Fibrinogen triggers astrocyte scar formation by promoting the availability of active TGF- $\beta$ after vascular damage. J Neurosci 30: 5843-5854, 2010.

85. Jeong SR, Kwon MJ, Lee HG, et al: Hepatocyte growth factor reduces astrocytic scar formation and promotes axonal growth beyond glial scars after spinal cord injury. Exp Neurol 233: 312-322, 2011.

86. Seth A and Watson DK: ETS transcription factors and their emerging roles in human cancer. Eur J Cancer 41: 2462-2478, 2005.

87. Powell RJ, Simons M, Mendelsohn FO, et al: Results of a double-blind, placebo-controlled study to assess the safety of intramuscular injection of hepatocyte growth factor plasmid to improve limb perfusion in patients with critical limb ischemia. Circulation 118: 58-65, 2008.

88. Shimamura M, Sato N, Yoshimura S, Kaneda Y and Morishita R: HVJ-based non-viral gene transfer method: successful gene therapy using HGF and VEGF genes in experimental ischemia. Front Biosci 11: 753-759, 2006. 
89. Bosch A, McCray Jr PB, Walters KS, et al: Effects of keratinocyte and hepatocyte growth factor in vivo: implications for retrovirus-mediated gene transfer to liver. Hum Gene Ther 9: 1747-1754, 1998.

90. Nolta JA and Kohn DB: Comparison of the effects of growth factors on retroviral vector-mediated gene transfer and the proliferative status of human hematopoietic progenitor cells. Hum Gene Ther 1: 257-268, 1990.

91. Culver KW, Ram Z, Wallbridge S, Ishii H, Oldfield EH and Blaese RM: In vivo gene transfer with retroviral vector-producer cells for treatment of experimental brain tumors. Science 256 1550-1552, 1992.

92. Thomas CE, Ehrhardt A and Kay MA: Progress and problems with the use of viral vectors for gene therapy. Nat Rev Genet 4: 346-358, 2003.

93.Zhao MZ, Nonoguchi N, Ikeda N, et al: Novel therapeutic strategy for stroke in rats by bone marrow stromal cells and ex vivo HGF gene transfer with HSV-1 vector. J Cereb Blood Flow Metab 26: 1176-1188, 2006.

94. Li JF, Yin HL, Shuboy A, et al: Differentiation of hUC-MSC into dopaminergic-like cells after transduction with hepatocyte growth factor. Mol Cell Biochem 381: 183-190, 2013.
95. Kurozumi K, Nakamura K, Tamiya T, et al: BDNF gene-modified mesenchymal stem cells promote functional recovery and reduce infarct size in the rat middle cerebral artery occlusion model. Mol Ther 9: 189-197, 2004.

96. Neuss S, Becher E, Wöltje M, et al: Functional expression of $\mathrm{HGF}$ and HGF receptor/c-met in adult human mesenchymal stem cells suggests a role in cell mobilization, tissue repair, and wound healing. Stem Cells 22: 405-414, 2004.

97. Ishikawa H, Jo JI and Tabata Y: Liver anti-fibrosis therapy with mesenchymal stem cells secreting hepatocyte growth factor. J Biomater Sci Polym Ed 23: 2259-2272, 2012

98. Gao CF and Woude GFV: HGF/SF-Met signaling in tumor progression. Cell Res 15: 49-51, 2005.

99. Zhang YW, Wang LM, Jove R and Vande WG: Requirement of Stat3 signaling for HGF/SF-Met mediated tumorigenesis. Oncogene 21: 217-226, 2002

100. Qian F, Engst S, Yamaguchi K, et al: Inhibition of tumor cell growth, invasion, and metastasis by EXEL-2880 (XL880, GSK1363089), a novel inhibitor of HGF and VEGF receptor tyrosine kinases. Cancer Res 69: 8009-8016, 2009.

101. Peruzzi B and Bottaro DP: Targeting the c-Met signaling pathway in cancer. Clin Cancer Res 12: 3657-3660, 2006. 